\title{
Exposición ocupacional a ruido y vibraciones en cuerpo entero en agricultores de la Zona Norte de Cartago, Costa Rica
} Occupational exposure to noise and whole-body vibration in farmers of the Northern Area of Cartago, Costa Rica

Tannia Araya-Solano' ${ }^{1}$ Ara Villalobos-Rodríguez ${ }^{2}$

Fecha de recepción: 24 de agosto de 2019 Fecha de aprobación: 18 de noviembre de 2019

Araya-Solano, T; Villalobos-Rodríguez, A. Exposición ocupacional a ruido y vibraciones en cuerpo entero en agricultores de la Zona Norte de Cartago, Costa Rica. Tecnología en Marcha. Vol. 33-4. Octubre-Diciembre 2020. Pág 3-14.

doi https://doi.org/10.18845/tm.v33i4.4470

1 MSc. Salud Ocupacional, Escuela de Ingeniería en Seguridad Laboral e Higiene Ambiental. Instituto Tecnológico de Costa Rica. Costa Rica. Correo electrónico: taraya@itcr.ac.cr. ne Ambiental. Instituto Tecnológico de Costa Rica. Costa Rica. Correo electrónico: avillalobos@itcr.ac.cr. 


\title{
Palabras clave
}

Vibraciones; agricultura; trabajadores agrícolas; vibraciones cuerpo entero; ruido; maquinaria agrícola.

\section{Resumen}

Como parte de las actividades que se realizan en el sector agrícola se hace uso de vehículos, por lo que se tiene contacto con diferentes agentes físicos como el ruido y las vibraciones de cuerpo entero, debido a esto el objetivo de este proyecto fue determinar la exposición ocupacional a estos agentes durante el uso del tractor, específicamente en labores realizadas en la Zona Norte de Cartago, Costa Rica. Para alcanzar dicho objetivo, se efectuó un estudio exploratorio en 13 fincas, en las cuales se cuantificaron los valores de exposición a ambos agentes, haciendo uso de un vibrómetro y audiodosímetros. Como resultado, se obtuvo que los niveles de exposición diaria a vibraciones se encuentran sobre el valor de acción en el $53,8 \%$ de los casos, y los resultados del nivel sonoro continuo equivalente (NSCE) se ubican entre los $54,8 \mathrm{~dB}(\mathrm{~A})$ a $75,4 \mathrm{~dB}(\mathrm{~A})$, por lo que no sobrepasan el nivel de exposición ocupacional. Además, se identificaron los factores de riesgo relacionados con estos agentes, la antigüedad de la maquinaria y dispositivos colocados en el vehículo, así como los factores personales que pueden influir en la aparición de dolencias, relacionadas con la actividad laboral. Existen otros factores de riesgo en la población en estudio, asociados a las posturas adoptadas y al índice de masa corporal de los colaboradores. Se concluye que la exposición a las vibraciones en cuerpo entero, podría llegar a generar efectos a la salud a largo plazo, además, el dispositivo de acople y modelo se convierten en factores que pueden influir en la exposición.

\section{Keywords}

Tractor; occupational exposure; whole body vibration; noise.

\begin{abstract}
The use of tractor in the agricultural sector is very common, the physical agents associated with it use are noise and vibrations of the whole body; the objective of this project was to determine occupational exposure to these agents during the use of the tractor in 13 small companies of the northern zone of Cartago, Costa Rica. The exposure were measurement with the ISO 26311: 2001 and noise dosimeter. As a result, it was obtained that daily vibration exposure levels are above the action level in $53,8 \%$ of cases, and the noise level is between $54,8 \mathrm{~dB}(\mathrm{~A})$ a $75,4 \mathrm{~dB}(\mathrm{~A})$, below occupational level exposure. The risks factors identified were related to the model and devices used in the equipment, postures and body mass index. The main conclusion is that the exposure to whole body vibration may cause health problems in the term, the model and devices used in the equipment are a risk factor of the exposure.
\end{abstract}

\section{Introducción}

La agricultura es uno de los sectores que presenta más peligros [1], generándose gran cantidad de lesiones y enfermedades relacionadas con pesticidas, problemas respiratorios, trastornos musculo esqueléticos, entre otros [2]. Las actividades que se realizan en este sector demandan una carga física muy alta, razón por la cual en los últimos años se ha aumentado la mecanización de la agricultura [3]. Sin embargo, la exposición a la maquinaria agrícola trae consigo otra serie de riesgos como las vibraciones de todo el cuerpo [4], así como el ruido [5], que si se llegan a convertir en excesivos pueden conducir a efectos crónicos y agudos en la salud [6]. 
Los diferentes implementos adaptados en la maquinaria para las labores de agricultura, generan variaciones en la exposición de las vibraciones de cuerpo entero [7]; además, la irregularidad del terreno y la velocidad del vehículo son factores importantes a considerar durante las evaluaciones [8].

El desarrollo de la tecnología en los procesos de mecanización agrícola ocasiona afectación en el entorno de trabajo para los usuarios; equipos como los tractores son de las fuentes más importantes de ruido [9]; además, factores como el manejo del equipo y el tipo de superficie perjudican la eficiencia de los operadores y por lo tanto el desempeño de los mismos [10].

Las vibraciones y el ruido se caracterizan porque se generan de la misma manera [11]; y ambos agentes se encuentran relacionados con los sistemas de transporte, pudiendo llegar a afectar la salud de muchas personas, por lo que el impacto en la calidad de vida puede ser importante [12].

El ruido puede definirse como un sonido no deseado [13] y es reconocido como una de las principales formas de contaminación, que causa diferentes daños como los auditivos, [14], siendo la pérdida de la audición una lesión común en los trabajadores agrícolas [15]; ocupación que se clasifica entre las primeras tres con mayor riesgo, debido al ruido excesivo durante las actividades laborales [16]. Además de la pérdida auditiva, otros de los efectos conocidos producto del ruido, son los efectos cardiovasculares [17], afectación en el sistema digestivo, respiratorio y en el metabolismo [18].

Por su parte, las vibraciones corresponden a oscilaciones de partículas alrededor de una posición de equilibrio que pueden generar desde falta de confort hasta fatiga [19], específicamente en las vibraciones de cuerpo entero la masa corporal se encuentra expuesta a vibraciones mecánicas, como las producidas por el asiento del tractor [20], ocasionando enfermedades cardiovasculares, respiratorias, afectación en el sistema endocrino, [21], siendo uno de los principales riesgos los trastornos musculo esqueléticos [22], que tienen efectos en la columna vertebral [23]; [24].

En la Unión Europea, un 23\% de los trabajadores agrícolas sufren dolores relacionados con el sistema musculo esquelético [1]. Específicamente en las actividades de conducción de vehículos, la edad, la amplitud de la vibración [21] y la carga postural, pueden contribuir a los trastornos relacionados con la espalda baja como las lumbalgias [25], [26].

El dolor lumbar es un importante problema de salud pública [27], encontrándose entre los que más costos generan para la sociedad en general [28]; la magnitud y la duración de la exposición a la vibración [29], son algunos de los factores que ocasionan esos dolores [30], por lo que los conductores de equipos como los tractores, que son utilizados durante muchas horas al día por los agricultores [31], se exponen a ese tipo de daño.

En general las actividades agrícolas presentan muchas limitaciones [32] que afectan la salud y seguridad de los empleados, por lo tanto es fundamental identificar los factores de riesgo, para luego desarrollar medidas adecuadas que permitan la gestión de las fuentes que los originan [33].

Por lo citado anteriormente y debido a que en Costa Rica no existen datos sobre la exposición a vibraciones y ruido en el sector agrícola, el objetivo de este proyecto fue determinar la exposición ocupacional a ruido y vibraciones en cuerpo entero durante el uso del tractor en agricultores de la zona norte de Cartago, Costa Rica, así como identificar si la antigüedad de la maquinaria, terreno, dispositivos de arrastre y velocidad, son factores que pueden influir en la exposición y si las posturas, edad, jornadas, y peso, son variables que pueden influir en la aparición de dolencias con el fin de iniciar investigaciones que mejoren las condiciones en este sector. 


\section{Materiales y métodos}

La investigación fue exploratoria, debido a que no se contaba con datos en el país sobre niveles de exposición a vibraciones en cuerpo entero y ruido durante el uso de tractores. La población de operarios de tractor en Tierra Blanca es un número que no se encuentra definido, por tanto, la selección de la muestra fue no representativa y a conveniencia. Se contactaron dueños de diferentes fincas ubicadas en la Zona Norte de Cartago pertenecientes a la Asociación de Horticultores del Irazú (AHSORI), como respuesta se obtuvo la participación de 13 fincas y 10 hombres adultos, debido a que uno de ellos prestó servicios de arado en tres diferentes fincas.

Vibraciones: Se siguió la metodología sugerida en la norma ISO 2631:2001: Exposición a vibraciones en cuerpo entero, para obtener los niveles de vibración a los que se exponen los trabajadores, utilizando el equipo HAV PRO, marca Quest Technologies tipo 1, serie número 07111, el cual cumple con los estándares ISO:8041:1990 y 1:1999(t), y se encontraba calibrado bajo condiciones de laboratorio. El sensor se colocó sobre el asiento del conductor respetando el sistema de coordenadas (X, Y, Z). Las mediciones se realizaron durante un tiempo aproximado de una hora, en cada caso evaluado, con una ponderación de exposición por minuto, por tanto, se generaron 60 datos por cada medición, las cuales fueron promediadas a un dato por cada 20 min. Las escalas de ponderación utilizadas fueron las establecidas para la evaluación de los efectos sobre la salud que se indican en la norma, con sus respectivos factores de multiplicación: Eje x: Wd, k=1.4; Eje y: Wd, k=1.4; Eje z: Wk, k=1.

Con los resultados obtenidos se calculó el factor cresta: rms/pico [34] para cada tractor. En los casos donde el factor cresta fue mayor a 9 se utilizó el método básico y el método alternativo Valor dosis de la vibración (VDV), que es más sensible a los picos de aceleración. Para cada eje se calculó el valor de exposición diaria A(8) y el valor de dosis según las siguientes ecuaciones [34]:

$$
\begin{gathered}
A_{x}(8)=1,4^{*} a_{w \times} \sqrt{\frac{T e x p}{T 0}}, \quad \operatorname{Ay}(8)=1,4^{*} a_{w \times} \sqrt{\frac{T e x p}{T 0}}, \quad A_{x}(8)=1,4^{*} a_{w x} \sqrt{\frac{T e x p}{T 0}} \\
\operatorname{VDV}_{x,}(8)=1,4^{*} V D V_{x}\left(T_{\text {exp }} / T_{\text {meas }}\right)^{1 / 4}, \operatorname{VDV}_{\mathrm{y},}(8)=1,4^{*} \operatorname{VDVy}\left(T_{\text {exp }} / T_{\text {meas }}\right)^{1 / 4}, \\
\operatorname{VDVz}(8)=\operatorname{VDVz}\left(T_{\text {exp }} / T_{\text {meas }}\right)^{1 / 4}
\end{gathered}
$$

Se tomó el eje crítico para realizar la evaluación del nivel de vibración. Además, los resultados se agruparon según el modelo del tractor. Para determinar la influencia de estos factores sobre la exposición diaria, se realizó una prueba de normalidad de los datos, usando el estadístico de prueba de Anderson-Darling, con un nivel de confianza del 95\%, a partir del programa estadístico Minitab 19. Posteriormente se planteó si hay una diferencia significativa entre la aceleración producida con el mecanismo de acople y sin él, así como el modelo del tractor, pues son variables pueden influir en la exposición ocupacional a vibraciones.

Para la comparación con la norma, se utilizó la Directiva Europea 2002/44/EC (2002) sobre Vibración Mecánica, debido a que Costa Rica no cuenta con límites de exposición para vibraciones. En la misma se establece para la exposición diaria valores límite de 1,15 m/s² y $0,5 \mathrm{~m} / \mathrm{s}^{2}$ de acción, y para el valor de dosis de la exposición $21 \mathrm{~m} / \mathrm{s}^{1.75}$ como límite y $9,1 \mathrm{~m} / \mathrm{s}^{1.75}$ como nivel de acción.

Ruido: Se realizaron audiodosimetrías con un dosímetro marca 3M, modelo Edge 4, aplicable a las normas ANSI S1.25-1991, ponderación A, ISO-1999, BS6402:1983. Con el porcentaje de dosis obtenido, se calculó el nivel sonoro continuo equivalente (NCSE), el cual se obtiene por medio de la siguiente fórmula:

$$
\text { NSCE }=\left[85+16,6 \log \left(\% \text { dosis } / 12,5{ }^{*} \mathrm{~T}\right)\right][35]
$$


Para cada medición, posteriormente se comparó con el nivel máximo permitido de exposición a ruido de $85 \mathrm{~dB}(\mathrm{~A})$ establecido en el Reglamento para el control de Ruido y Vibraciones de Costa Rica [36].

Determinantes de exposición y factores de riesgo: La identificación de factores de riesgo que pueden influir en la aparición de dolencias, se realizó, recopilando información a partir del Cuestionario para vigilancia de la Salud por Vibración de Cuerpo entero: EC Biomed II BMH4CT98-3251_[37] con respecto al tiempo de exposición, peso, edad, altura, dolencias percibidas en la espalda, cuello y hombros, así como su intensidad en los últimos 12 meses. Con el dato del peso y altura se procedió a calcular el índice de masa corporal (IMC), que se obtiene de la siguiente ecuación [38]:

$$
\mathrm{IMC}=\text { Peso }(\mathrm{kg}) \div \text { altura }\left(\mathrm{m}^{2}\right)
$$

\section{Resultados}

\section{Datos generales}

El estudio exploratorio contó con la participación de un colaborador conductor de tractor, por cada una de las 13 fincas dedicadas a la agricultura, ubicadas en la Zona Norte de Cartago. La edad de los conductores se encuentra entre los 21 y 56 años, quienes tienen de 5 a 34 años de experiencia en el manejo de tractores. La jornada laboral varía entre 8 a 10 horas de trabajo. Las horas de exposición a las vibraciones y ruido son de 5 o 9 horas, dependiendo de la empresa y del tipo de trabajo que esté realizando.

\section{A. Vibraciones}

El sensor del vibrómetro se colocó en el asiento del conductor, respetando los ejes (x,y,z). Se calculó el factor cresta para cada uno de los datos recopilados; en todos los casos resultó por encima de nueve, por lo que se utilizaron tanto el método básico como el VDV, según lo establecido en la ISO 2631-1 [39].

En el cuadro 1 se muestran los datos obtenidos de aceleración en cada una de las mediciones realizadas. El terreno sobre el cual se trabajó era tierra.

Como se muestra en el cuadro 1, en el $31 \%$ de los casos predomina el eje $\mathrm{X}$, el $62 \%$ el eje $\mathrm{Y}$ y en el $7 \%$ en el eje Z, lo que se podría indicar que los movimientos laterales y de frente hacia atrás son los más frecuentes, esto se puede deber a los movimientos producidos por las tareas a realizar, así como irregularidad del terreno o presencia de piedras en el mismo. Adicionalmente, en el 53,8\% casos se sobrepasa el nivel de acción $\left(0,5 \mathrm{~m} / \mathrm{s}^{2}\right)$, y en el $15 \%$ de los casos sobrepasa el límite recomendado $\left(1,15 \mathrm{~m} / \mathrm{s}^{2}\right)$. El $68.8 \%$ de los colaboradores se encuentra en riesgo de presentar afecciones a la salud, producto de la exposición a vibraciones. Según una investigación realizada en campo [40] con tractores nuevos, prácticamente todos ( 95\%) excedieron el Valor de Acción de Exposición (EAV) durante un día de 8 horas.

El valor de dosis de la vibración se encontró entre 0,0314 m/s $\mathrm{s}^{1 / 4}$ y 573,0153 m/s $\mathrm{s}^{1 / 4}$, los ejes predominantes son el $\mathrm{X}$ y $\mathrm{Y}$, con un $38 \%$ de los datos cada uno, coincidiendo con los ejes predominantes del cuadro 1. Se estimó el valor diario de la exposición y se comparó con los valores límites, resultando que el $46 \%$ de todas las mediciones se encuentran sobre el valor de acción. Debido a los movimientos producidos durante el uso del implemento y las irregularidades en el terreno, en algunos casos como la medición 9,10 y 12 no se puede definir un eje predominante, debido a que los valores son muy similares. 
Cuadro 1. Valores de aceleración promedio en $\mathrm{m} / \mathrm{s}^{2}$, de la exposición diaria A (8) promedio en $\mathrm{m} / \mathrm{s}^{2}$, obtenidos de cada una de las mediciones $(n=13)$.

\begin{tabular}{|c|c|c|c|c|c|c|}
\hline № medición & $\begin{array}{l}\text { Aeq X } \\
\left(\mathrm{m} / \mathrm{s}^{2}\right)\end{array}$ & $\begin{array}{l}\text { Aeq Y } \\
\left(\mathrm{m} / \mathrm{s}^{2}\right)\end{array}$ & $\begin{array}{l}\text { Aeq Z } \\
\left(\mathrm{m} / \mathrm{s}^{2}\right)\end{array}$ & $\begin{array}{l}A(8) x \\
\left(\mathrm{~m} / \mathrm{s}^{2}\right)\end{array}$ & $\begin{array}{l}A(8) y \\
\left(\mathrm{~m} / \mathrm{s}^{2}\right)\end{array}$ & $\begin{array}{l}A(8) z \\
\left(\mathrm{~m} / \mathrm{s}^{2}\right)\end{array}$ \\
\hline $1^{*}$ & 0,5744 & 0,8338 & 0,5523 & 0,6357 & 0,9229 & 0,4367 \\
\hline $2^{\star \star}$ & 0,3645 & 0,4061 & 0,3294 & 0,5413 & 0,6030 & 0,3493 \\
\hline $3^{* *}$ & 0,0517 & 0,0882 & 1,2270 & 0,0767 & 0,1310 & 1,3014 \\
\hline $4^{\star \star}$ & 0,1493 & 0,4159 & 0,4788 & 0,2278 & 0,6345 & 0,5218 \\
\hline $5^{\star \star}$ & 0,0863 & 0,0978 & 0,0478 & 0,1281 & 0,1452 & 0,0507 \\
\hline $6^{\star *}$ & 0,0020 & 0,0029 & 0,0067 & 0,0030 & 0,0043 & 0,0071 \\
\hline $7^{\star \star}$ & 0,3798 & 0,3355 & 00,3720 & 0,5318 & 0,4697 & 0,3792 \\
\hline $8^{*}$ & 0,1910 & 0,1692 & 0,1929 & 0,2835 & 0,2512 & 0,2046 \\
\hline $9^{*}$ & 1,1087 & 1,1310 & 0,4590 & 1,6463 & 1,6795 & 0,4869 \\
\hline $10^{*}$ & 0,3819 & 0,4136 & 0,3177 & 0,5346 & 0,5790 & 0,3177 \\
\hline $11^{*}$ & 0,4718 & 0,2590 & 0,2679 & 0,7005 & 0,3846 & 0,2842 \\
\hline $12^{*}$ & 0,2240 & 0,2491 & 0,2055 & 0,3326 & 0,3699 & 0,2180 \\
\hline $13^{* \star}$ & 0,4078 & 0,3867 & 0,2715 & 0,6056 & 0,5742 & 0,2879 \\
\hline
\end{tabular}

Nota: Aeq X: aceleración equivalente en el eje X, Aeq Y: aceleración equivalente en el eje Y, Aeq Z: aceleración equivalente en el eje Z; A (8)x: aceleración de la exposición diaria en el eje x; A(8)y: aceleración de la exposición diaria en el eje y; A(8)z: aceleración de la exposición diaria en el eje z. *acople con arado, **acople con rotador.

Otras investigaciones reflejan que durante el uso del tractor es el eje Z el predominante [41], sin embargo, las condiciones del terreno fueron diferentes. También se reportaron como eje predominante el Y, con valores del VDV de $16.6 \mathrm{~m} / \mathrm{s}^{1 / 4}$, sobrepasando el nivel de acción [42], coincidiendo así con lo calculado en el cuadro 2.

En el cuadro 3 se muestran los datos de aceleración agrupados por modelo.

Como se puede observar en el cuadro anterior, la exposición diaria en el 7\% de los casos está por debajo del nivel de acción, específicamente para el modelo 2005, otro 62\% está sobre el nivel de acción (modelos 2008, 2010, 2011, 2015), y el porcentaje restante 31\% se encuentra sobre el valor límite de exposición diaria de 1,15m/s² (modelos 2007 y 2013). Por lo tanto, existe riesgo para la salud de los conductores.

En el caso del VDV, el 31\% de los valores de exposición diaria, están por debajo del nivel de acción (modelos 2005, 2013 y 2015), el 54\% sobre el valor de acción (modelos 2008, 2010, 2011) y el 14\% (modelo 2007) por encima del nivel límite de exposición, esto se debe a que en ocasiones el arado de disco o rotador chocaba con piedras que se encontraban en el terreno. 
Cuadro 2. Valores de aceleración, valor de dosis de la vibración promedio para cada eje, exposición diaria VDV (8) promedio en $\mathrm{m} / \mathrm{s}^{1 / 4}$, y valor de dosis diaria $\left(\mathrm{m} / \mathrm{s}^{1 / 4}\right)(\mathrm{n}=13)$

\begin{tabular}{|c|c|c|c|c|c|c|}
\hline$N^{\circ}$ medición & $\begin{array}{c}\text { VDVx } \\
(\mathrm{m} / \mathrm{s} 1 / 4)\end{array}$ & $\begin{array}{c}\text { VDVy } \\
(\mathrm{m} / \mathrm{s} 1 / 4)\end{array}$ & $\begin{array}{c}\text { VDVz } \\
(\mathrm{m} / \mathrm{s} 1 / 4)\end{array}$ & $\begin{array}{c}\text { VDV }(8) x \\
(\mathrm{~m} / \mathrm{s} 1 / 4)\end{array}$ & $\begin{array}{c}\text { VDV (8)y } \\
(\mathrm{m} / \mathrm{s} 1 / 4)\end{array}$ & $\begin{array}{c}\text { VDV (8)z } \\
(\mathrm{m} / \mathrm{s} 1 / 4)\end{array}$ \\
\hline 1 & 4,5279 & 6,5356 & 5,8638 & 8,2415 & 11,8958 & 7,6237 \\
\hline 2 & 3,3014 & 3,7336 & 6,0761 & 8,3787 & 9,4758 & 11,0150 \\
\hline 3 & 1,2120 & 1,5128 & 36,8836 & 2,4713 & 3,0848 & 53,7201 \\
\hline 4 & 1,2435 & 3,3956 & 3,4747 & 4,1299 & 11,2771 & 8,2426 \\
\hline 5 & 1,6970 & 1,9410 & 0,8796 & 4,8935 & 5,5973 & 1,8118 \\
\hline 6 & 0,0443 & 0,0512 & 0,0799 & 0,1415 & 0,1634 & 0,1821 \\
\hline 7 & 3,0785 & 2,6133 & 2,9401 & 9,5395 & 8,0977 & 6,5076 \\
\hline 8 & 1,5614 & 1,3322 & 1,5100 & 4,1902 & 3,5751 & 2,8944 \\
\hline 9 & 3,5619 & 3,5824 & 3,3708 & 7,9642 & 8,0100 & 5,3835 \\
\hline 10 & 3,0511 & 3,0327 & 2,5036 & 9,4543 & 9,3974 & 5,5415 \\
\hline 11 & 2,2312 & 2,2956 & 4,6800 & 5,9874 & 6,1604 & 8,9707 \\
\hline 12 & 2,0094 & 1,9746 & 2,1616 & 5,3922 & 5,2990 & 4,1434 \\
\hline 13 & 3,6367 & 2,9741 & 2,9584 & 8,4852 & 6,9391 & 4,9303 \\
\hline
\end{tabular}

Nota: VDVx: valor de dosis de la vibración en el eje x, VDVy: valor de dosis de la vibración en el eje y, VDVz: valor de dosis de la vibración en el eje z, VDV(8)x: valor de dosis de la vibración diaria en el eje x, VDV(8)y: valor de dosis de la vibración diaria en el eje y, VDV(8)z: valor de dosis de la vibración diaria en el eje z.

Cuadro 3. Valores de aceleración, valor de dosis de la vibración promedio para cada eje, exposición diaria A (8) promedio en $\mathrm{m} / \mathrm{s}^{2}$, y valor de dosis diaria $\left(\mathrm{m} / \mathrm{s}^{1 / 4}\right)$ agrupados por modelo del tractor $(n=13)$.

\begin{tabular}{|c|c|c|c|c|c|c|}
\hline $\begin{array}{c}\text { Modelo } \\
\text { (cantidad } \\
\text { tractores) }\end{array}$ & $\begin{array}{c}\mathrm{A}(8) \mathrm{x} \\
\left(\mathrm{m} / \mathrm{s}^{2}\right)\end{array}$ & $\begin{array}{c}\mathrm{A}(8) \mathrm{y} \\
\left(\mathrm{m} / \mathrm{s}^{2}\right)\end{array}$ & $\begin{array}{c}\mathrm{A}(8) z \\
\left(\mathrm{~m} / \mathrm{s}^{2}\right)\end{array}$ & $\begin{array}{c}\text { VDV }(8) x \\
\left(\mathrm{~m} / \mathrm{s}^{1 / 4}\right)\end{array}$ & $\begin{array}{c}\text { VDV }(8) y \\
\left(\mathrm{~m} / \mathrm{s}^{1 / 4}\right)\end{array}$ & $\begin{array}{c}\text { VDV }(8) z \\
\left(\mathrm{~m} / \mathrm{s}^{1 / 4}\right)\end{array}$ \\
\hline $2005(1)$ & 0,0030 & 0,0043 & 0,0071 & 0,1415 & 0,1634 & 0,1821 \\
\hline $2007(1)$ & 0,0767 & 0,1310 & 1,3014 & 2,4713 & 3,0848 & 53,7201 \\
\hline $2008(2)$ & 0,5186 & 0,7434 & 0,3476 & 7,4689 & 10,4423 & 6,2825 \\
\hline $2010(2)$ & 0,4965 & 0,6075 & 0,3740 & 7,7718 & 9,7331 & 10,6189 \\
\hline $2011(3)$ & 0,5318 & 0,4697 & 0,3792 & 9,5395 & 8,0977 & 6,5076 \\
\hline $2013(3)$ & 1,0981 & 1,1142 & 0,3821 & 7,0988 & 6,9411 & 4,6949 \\
\hline $2015(1)$ & 0,5581 & 0,4691 & 0,2683 & 6,9943 & 6,2941 & 5,7979 \\
\hline
\end{tabular}

\section{A. 1. Influencia de dispositivo de acople y modelo del tractor en la exposición.}

Los dispositivos utilizados, como se mencionó anteriormente, fueron arados de disco y rotador; el terreno fue plano o inclinado; los modelos evaluados fueron 2005, 2007, 2008, 2010, 2011 , 2013 y 2015; la velocidad fue un dato que no se pudo recolectar, debido a que variaba según el terreno. 
Para determinar si el modelo del tractor y el dispositivo de acople influyen en la exposición a vibraciones, se realizó una ANOVA.

Como resultado se obtuvo que, en la comparación entre modelos, el valor de $\mathrm{P}=0.000$, por lo tanto, si hay suficiente evidencia estadística para concluir que el modelo si influye en la exposición.

En cuanto al dispositivo de acople el valor de $P=0.000$, esto sugiere que hay suficientemente evidencia estadística para concluir que el acople influye en la exposición a la vibración.

\section{B. Ruido}

Para el caso de ruido, se tomaron solo 9 muestras, ya que en cuatro ocasiones al realizarse la visita estaba lloviendo, por lo que no se pudo colocar el dosímetro. Una vez que se obtuvieron los valores de porcentaje de dosis, éstos se proyectaron a 8 horas, partiendo del supuesto de que el nivel de exposición es constante, basado en que las condiciones generadoras de ruido no cambian durante la jornada. Luego se realizó el cálculo del nivel sonoro continuo equivalente.

Los valores del NSCE se encuentran en entre los 54,8dB(A) a 75,4 dB(A), los mismos se muestran en la siguiente figura 1.

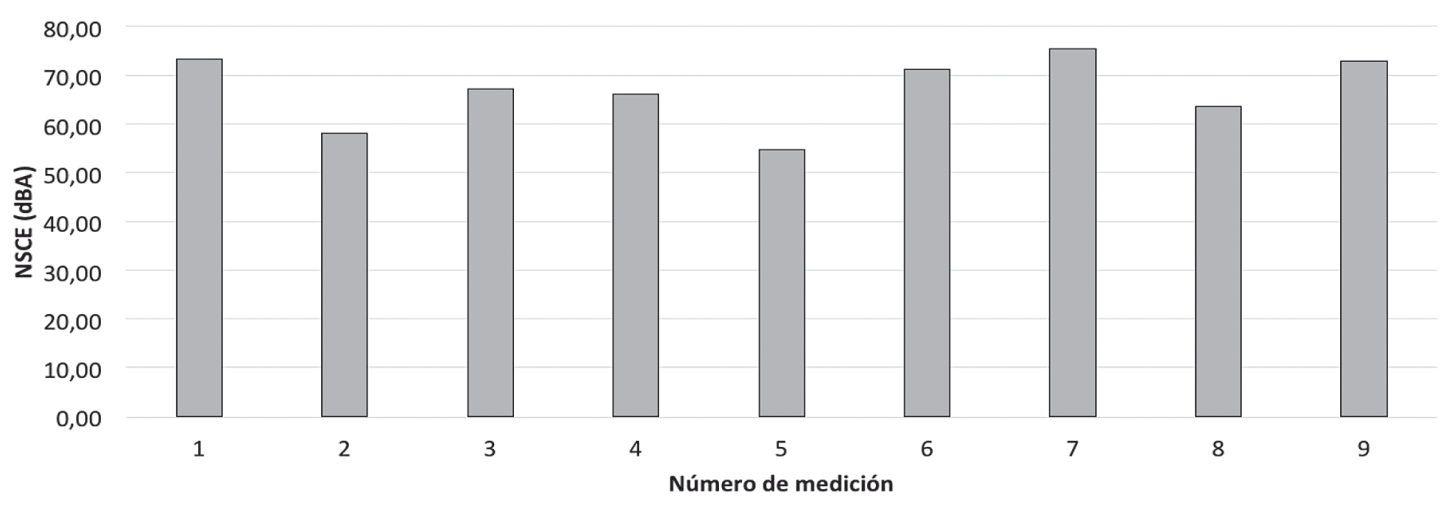

Figura 1. Nivel sonoro continuo equivalente, en dB(A), para cada medición realizada.

Como se puede observar en la figura anterior, ninguno de los datos sobrepasa el valor recomendado de $85 \mathrm{~dB}(\mathrm{~A})$, establecido en la legislación costarricense. De acuerdo con las investigaciones consultadas de otros países [43], durante el uso de los tractores en labores agrícolas se obtienen niveles entre $62,1 \mathrm{~dB}(\mathrm{~A})$ a $87,4 \mathrm{~dB}(\mathrm{~A})$ y promedios de $68,2 \mathrm{~dB}(\mathrm{~A})$ a 83,8 $\mathrm{dB}(\mathrm{A})$.

\section{Determinantes de exposición y factores de riesgo}

Se entrevistó a nueve colaboradores, debido a que una de las personas participantes se negó a brindar la información. Los participantes cuentan con experiencia entre los 5 y 34 años en conducción de tractores; adicionalmente, todos han laborado con maquinaria pesada 0 camiones; a ellos se les aplicó el Cuestionario para vigilancia de la salud por vibración de cuerpo entero: EC Biomed. Las posturas que se adoptan en las tareas es inclinada hacia adelante y retorcido (viendo hacia atrás).

A continuación, se muestra el porcentaje de las partes del cuerpo, asociadas a la actividad evaluada, donde se presenta mayor dolencia externada por los trabajadores, según la frecuencia: 


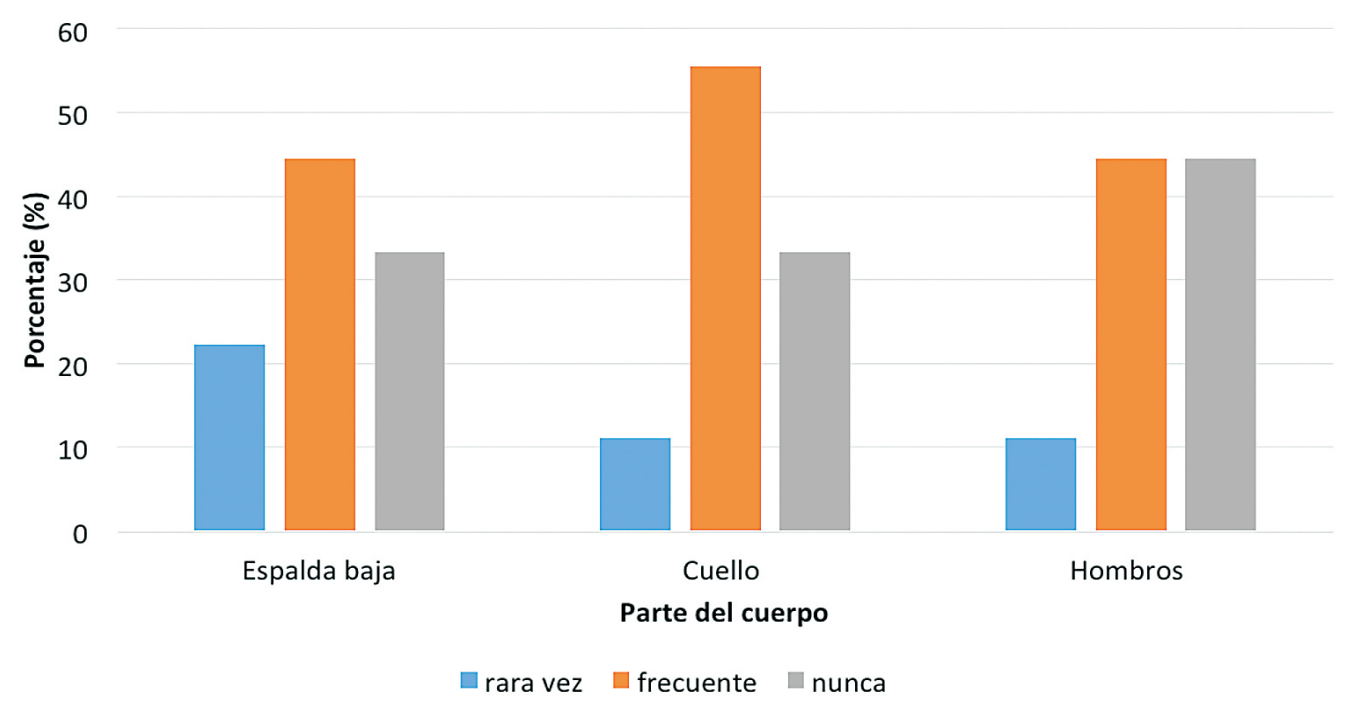

Figura 2. Porcentaje de las partes del cuerpo donde se presentan dolencias.

La parte del cuerpo donde se presenta mayor porcentaje de dolencia es en el cuello; esto se puede deber a las posturas que se adoptan, debido a que tienen que estar volteando hacia atrás, para observar el dispositivo de arrastre colocado al tractor.

La intensidad del dolor en esta parte del cuerpo, es calificada por el $44 \%$ de las encuestados como muy significativa (nivel de 8 , en escala de 10), para espalda y para hombros es de 8 en el 33\% de los casos. Estas dolencias provocaron que 1 de los colaboradores se incapacitara por 7 días, en el último año.

Se han realizado encuestas en otros países donde los conductores de tractores manifiestan que el dolor de espalda es el más frecuente en su actividad laboral [4].

De acuerdo a la consulta efectuada sobre el peso de los trabajadores, se procedió a realizar el cálculo del índice de masa corporal (IMC), el cual en promedio es de 27; obteniéndose que la población en estudio se encuentra en condiciones de peso excesivo, muy cerca del valor de obesidad [38]; este trastorno puede ocasionar serias consecuencias para la salud, como lo es la aparición de varias enfermedades crónicas [44], como la hipertensión, diabetes, entre otras [45]. Siendo las enfermedades cardiovasculares la primera causa de muerte en Costa Rica, esto de acuerdo a un estudio realizado para la provincia de Cartago [46].

Además, otro factor que es importante considerar es la edad de los trabajadores, debido a que enfermedades como la presión arterial aumentada, tienen una mayor incidencia en personas de mayor edad [47]; en el caso específico de la población en estudio la edad promedio es de 39 años; sin embargo se debe considerar que uno de los trabajadores de mayor edad, era quien tenía uno de los IMC más altos, específicamente 31,1, catalogado por Mahan \& Escott_Stump (2009) como obeso; convirtiéndose en uno de los factores de riesgo para la salud [48].

\section{Conclusiones y/o recomendaciones}

Existe riesgo a la salud de los colaboradores, puesto que tanto los niveles de exposición diaria a vibraciones como el valor de dosis de la vibración, se encuentran sobre el nivel de acción, esto en el 53,8\% de los casos. En cuanto a la exposición a ruido, en todos los casos el NSCE se encuentra por debajo de los $85 \mathrm{~dB}(\mathrm{~A})$. Con los datos recopilados se concluye que hay evidencia estadística de que el dispositivo de acople y el modelo son factores que influyen en la 
exposición a vibraciones. Las dolencias a nivel de cuello son las más frecuentes; relacionadas con las torsiones en el tronco y cuello del colaborador al realizar la tarea. El IMC promedio, evidencia que existe un factor de riesgo a la salud de los colaboradores. Se recomienda establecer relaciones con representantes de cámaras, para que se logre obtener mayor respuesta e impacto en la presentación de los resultados. Sería prudente incluir dentro del equipo de trabajo, a un especialista en fisioterapia, con el fin de que colabore en la detección de las afecciones a la salud, relacionadas con la actividad laboral; además que elabore una propuesta de ejercicios compensatorios, que mejoren las condiciones actuales de este sector. Para la generación de nuevas investigaciones se recomienda ampliar el número de muestra y el tiempo de observación, para determinar la evolución de las dolencias y poder relacionarlas con las vibraciones. También trabajar con otros sectores, factor que a largo plazo permita impactar en mayor medida a la población.

\section{Agradecimientos}

A la Vicerrectoría de Investigación y Extensión del Tecnológico de Costa Rica, por el apoyo para la realización de este proyecto. A la Asociación de Horticultores del Irazú (AHSORI), quienes hicieron posible este proyecto con la oportunidad brindada. Adicionalmente se agradece a la asistente de la investigación la Señorita Geisel Segura.

\section{Referencias}

[1] C. Lunner Kolstrup, «Work-related musculoskeletal discomfort of dairy farmers and employed workers, Journal of Occupational Medicine and Toxicology, pp. 1-9, 2012.

[2] F. A. Fathallah, «Musculoskeletal disorders in labor-intensive agriculture,» Applied Ergonomics, pp. 738-743, 2010.

[3] A. Papageorgiou, «Agricultural equipment in Greece: Farm machinery management in the era of economic crisis,» Agriculture and Agricultural Science Procedia, pp. 198-202, 2015.

[4] K. Walker Bone y K. Palmer, «Musculoskeletal disorders in farmers and farm workers, Occupational Medicine, pp. 441-450, 2002.

[5] M. Lashgaria y A. Maleki, «Evaluation of lawn tractor noise using acoustic and psychoacoustic descriptors, » Engineering in Agriculture, Environment and Food, pp. 116-122, 2016.

[6] A. Verma, P. Guru y R. Naik, «Noise and Vibration Analysis of Power Tillers during Field Operations, International Journal of Bio-Resource \& Stress Management, pp. 288-293, 2014.

[7] T. H. Langer, M. K. Ebbesen y . A. Kordestani, «Experimental analysis of occupational whole-body vibration exposure of agricultural tractor with large square baler,» International Journal of Industrial Ergonomics, pp. 79-83, 2015.

[8] M. Cutini, . C. Costa y C. Bisaglia , «Development of a simplified method for evaluating agricultural tractor's operator whole body vibration, » Journal of Terramechanics , pp. 23-32, 2016.

[9] B. Bilski, «Exposure to audible and infrasonic noise by modern agricultural tractors operators, Applied Ergonomics, pp. 2010-2014, 2013.

[10] A. Aybek, H. A. Kamer y S. Arslan, «Personal noise exposures of operators of agricultural tractors,» Applied Ergonomics, pp. 274-281, 2010.

[11] M. Rikondo Iriondo, «Vibraciones mecánicas. Qué son y cómo se evalúa el riesgo?,» Seguridad Industrial, pp. 499-506, 2007.

[12] M. Kutz, Handbook of Transportation Engineering, Volume II: Applications and Technologies, New York: McGraw-Hill Education, 2011.

[13] K. B. Zandin, Maynard's Industrial Engineering Handbook, Fifth Edition, New York: McGRAW-HILL, 2001.

[14] R. A. Corbitt, Standard Handbook of Environmental Engineering, New York: McGRAW-HILL, 1999.

[15] W. Williams, S. Brumby, A. Calvano, T. Hatherell, H. Mason, C. Mercer-Grant y A. Hogan, «Farmers' work-day noise exposure, „ Australian Journal of Rural Health, pp. 67-73, 2015. 
[16] J. J. Ehlers y P. S. Graydon, «Noise-induced hearing loss in agriculture: Creating partnerships to overcome barriers and educate the community on prevention,» Noise \& Health, pp. 142-146, 2011.

[17] G. Tomei, A. Sancini, F. Tomei, A. Vitarelli, G. Andreozzi, . G. Rinaldi, V. Di Giorgio, . I. Samperi, M. Fiaschet, Z. Tasciotti, C. Cetica, . A. Capozzella, . M. Ciarrocca y T. Caciari, «Prevalence of Systemic Arterial Hypertension, Electrocardiogram Abnormalities, and Noise-Induced Hearing Loss in Agricultural Workers,» Archives of Environmental \& Occupational Health, pp. 196-202, 2013.

[18] J. M. Cortés Díaz, Seguridad e Higiene del Trabajo, Madrid: TÉBAR, 2012.

[19] R. Fernández García, "Las vibraciones. Una amenaza silenciosa para su salud,» Gestión práctica de riesgos laborales, pp. 7-12, 2015.

[20] J. V. Grimaldi y R. H. Simonds, La seguridad industrial, su adminitración, 16 ed., México: Alfaomega, 2009.

[21] R. Wolfgang y R. Burgess-Limerick, «Whole-body vibration exposure of haul truck drivers at a surface coal,» Applied Ergonomics, pp. 1700-1704, 2014.

[22] F. Caffaro, M. Micheletti Cremasco, . C. Preti y E. Cavallo, «Ergonomic analysis of the effects of a telehandler's active suspended cab on whole body vibration level and operator comfort, International Journal of Industrial Ergonomics, pp. 19-26, 2016.

[23] F. d. C. Acuña Vidal, y A. Dosil Caamaño, «Evaluación por estimación del riesgo de exposición a vibraciones mecánicas: Caso práctico,» Gestión práctica de riesgos laborales, pp. 24-31, 2015.

[24] X. Zhao y C. Schindler, «Evaluation of whole-body vibration exposure experienced by operators of a compact wheel loader according to ISO 2631-1:1997 and ISO 2631-5:2004,» International Journal of Industrial Ergonomics, pp. 840-850, 2014.

[25] Health \& Safety Executive, «Whole-body vibration and shock: A literature review,» 2001.

[26] M. Bovenzi, I. Pinto y N. Stacchini, «Low back pain in port machinery operators., Journal of sound and vibration, 253 (1), pp. 3-20, 2002.

[27] K. M. Black, . A. Lis y M. Nordin, «Association Between Sitting and Occupational Low Back Pain (LBP),» Ação Ergonômica, pp. 73-86.

[28] C. Hulshof, J. Verbeek, I. Braam, M. Bovenzi y F. van Dijk, «Evaluation of an occupational health intervention programme on whole-body vibration in forklift truck drivers: a controlled trial,» Occupational \& Environmental Medicine, pp. 461-468, 2006.

[29] L. Solecki, «Low back pain among farmers exposed to whole body vibration: a literature review,» Medycyna pracy, pp. 187-202, 2011.

[30] S. A. Adam y N. A. Abdul Jalil, «Vertical Suspension Seat Transmissibility and SEAT Values for Seated Person Exposed to Whole-body Vibration in Agricultural Tractor Preliminary Study,» Procedia Engineering, pp. 435442, 2017.

[31] R. Deboli , A. Calvo y C. Preti, «Whole-body vibration: Measurement of horizontal and vertical,» International Journal of Industrial Ergonomics, pp. 69-78, 2017.

[32] C. Chisholma, D. Bottoms, M. Dwyer, J. Lines y R. Whyte, «Safety, health and hygiene in agriculture,» Safety Science, pp. 225-248, 1992.

[33] M. Cecchini, R. Bedini, D. Mosetti, S. Marino y S. Stasi, "Safety Knowledge and Changing Behavior in Agricultural Workers: an Assessment Model Applied in Central Italy,» Safety and Health at Work, pp. 1-8, 2017.

[34] ISO, "Mechanical vibration and shock-Evaluation of exposure to whole body vibration. Part 1:General Requeriments. ISO2631-1:1997.,» 1997.

[35] F. Henao Robledo, Riesgos físicos I: Ruido, vibraciones y presiones anormales, Bogotá: ECOE Ediciones, 2008.

[36] MTSS, Reglamento para el Control de Ruidos y Vibraciones. Decreto No. 10541-TSS, San José, 1997.

[37] Vibration Injurie Network, "Guidelines and Questionnaires for Whole-Body Vibration Health Surveillance. Appendix W1A to Final Report,» 2001.

[38] L. K. Mahan y S. Escott-Stump, Krause Dietoterapia, Barcelona: ELSEVIER MASSON, 2009.

[39] International Standard Organization, Mechanical vibration and shock- Evaluation of exposure to whole body vibration. Part 1: General Requirements. ISO 2631-1:1997., 1997.

[40] A. Scarlett, D. Semple, J. Price y R. Stayner, «Whole-body vibration on agricultural vehicles: evaluation of emission and estimated exposure levels,» Journal of terramechanics, 44(1), pp. 65-73., 2005. 
[41] M. Raoufat, S. Hassanian y D. Vahid, «Whole-body vibrations, evaluation of emissions and exposure levels arising from a medium hp farm tractor., ") Tarim Makinalari Bilimi Dergisi, 7(2), 2011.

[42] S. Milosavljevic, F. Bergman, B. Rehn y A. Carman, «All-terrein vehicule use in agriculture: Exposure to whole body vibration and mechanical shock.," Applied ergonomic, 41(4), pp. 530-535, 2010.

[43] B. BILSKI, «AUDIBLE AND INFRASONIC NOISE LEVELS IN THE CABINS OF MODERN AGRICULTURAL TRACTORS - DOES THE RISK OF ADVERSE, EXPOSURE-DEPENDENT EFFECTS STILL EXIST?,» International Journal of Occupational Medicine and Environmental Health, pp. 488-493, 2013.

[44] C. H. Daza, «La obesidad: un desorden metabólico de alto riesgo para la salud,» Colombia Médica, vol. 33, $\mathrm{n}^{\circ}$ 2, pp. 72-80, 2002.

[45] A. Zugasti Murillo y B. Moreno Esteban, «Obesidad como factor de riesgo cardiovascular,» Hipertensión y riesgo vascular, vol. 22, $\mathrm{n}^{\circ}$ 1, pp. 32-36, 2005.

[46] W. I. Hernández Montoya, «Factores de riesgo de enfermedad cardiovascular en una población obrera industrial de la provincia de Cartago,» Revista Costarricense de Salud Pública, vol. 9, n 16, pp. 1409-1429, 2000.

[47] I. Palomo G, G. Icaza N, V. Mujica E, L. Núñez F, E. Leiva M, M. Vásquez R, M. Alarcón L y E. Moyano D, «Prevalencia de factores de riesgo cardiovascular clásicos en población adulta de Talca, Chile, 2005,» Revista médica de Chile, vol. 135, n 7, pp. 904-912, 2007.

[48] G. M. Moreno, "Definición y clasificación de la obesidad.," Revista Médica Clínica Las Condes, 23(2), pp. 124-128., 2012. 